\title{
Image Alignment in Pose Variations of Human Faces by using Corner Detection Method and its Application for PIFR System
}

\section{Deepika Dubey}

Uttarakhand Technical University

Geetam Singh Tomar ( $\nabla$ gstomar@ieee.org )

Birla Institute of Applied Sciences https://orcid.org/0000-0002-0246-1527

\section{Research}

Keywords: Multiscale Corner Detection, Neural Network (NN), Image Alignment, Pose Invariant Face Recognition (PIFR), Harris Corner Detection

Posted Date: January 4th, 2021

DOI: https://doi.org/10.21203/rs.3.rs-136431/v1

License: (c) (i) This work is licensed under a Creative Commons Attribution 4.0 International License. Read Full License

Version of Record: A version of this preprint was published at Wireless Personal Communications on November 19th, 2021. See the published version at https://doi.org/10.1007/s11277-021-09330-1. 


\title{
Image Alignment in Pose Variations of Human Faces by using Corner Detection Method and its Application for PIFR System
}

\author{
${ }^{1}$ Deepika Dubey, ${ }^{2}$ Geetam Singh Tomar \\ ${ }^{1}$ Department of Computer Science \& Engineering, Uttrakhand Technical University, Dehradun-248007, India \\ 2*Department of Electronics \& Communication Engineering, Birla Institute of Applied Sciences, Bhimtal -263136, India \\ E-mail: ${ }^{1}$ deepika.sa1304@gmail.com, ${ }^{2}$ gstomar@ieee.org * corresponding Author
}

\begin{abstract}
The Major challenge in the recent face recognition techniques is to deal with pose variations during matching as facial image differences occurs due to motion/rotation in image, which is very large. The Pose Invariant Face Recognition is still an open area for developers to find solution. In this paper focus is on PIFR techniques and combined it with other algorithms for enhancing the results. Here we are using the Harris Corner Detection model along with Image alignment and Image tagging to get front face images. By generalization different tricks to handle the pose on face images has minimized the pose variation. On evaluating performance of the system, we have also calculate the Euler angle and their position change and according to it correct the pose variation. The results are in accordance with the expected lines.
\end{abstract}

Keywords: Multiscale Corner Detection, Neural Network (NN), Image Alignment, Pose Invariant Face Recognition (PIFR), Harris Corner Detection

\section{Introduction}

The face recognition has been a wider area to work on the new techniques due to its applications in all aspects of life. This has prompted us to find further improvements in the techniques and approaches suggested in the available literature, which are basically using either the model-based technique or they are the appearancebased techniques. The output of face recognition is based on some physical factors like type of lightning conditions, rotation, contrast, expression etc. On the basis of these factors the input data could to the system changes considerably. Once the system gets the input, it starts processing the image using recognition algorithms to identify the facial features by identifying the landmarks on the input image to recognize the face with a face saved in its database $[2,3]$. Once the system identifies the landmarks on the subject image using different algorithms, it starts matching the basic features identified from the subject image with the images saved in its database to extract an image which matches with the subject image. Fig 1 shows basic structure of face detection and face recognition with identification and variation of faces. Face recognition is based on face detection as well as extraction process of face from image.

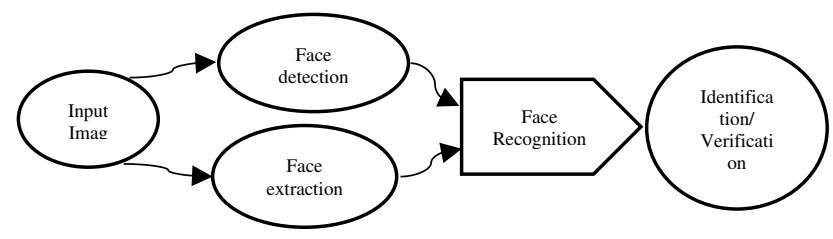

Fig.1: Face Detection and Recognition with Variation of faces

Face recognition techniques are being used as a mandatory feature for functioning of their systems. Let's take an example of social media in which face recognition has become a mandatory part for its functioning for example There are many apps using face recognition techniques to provide face modification tools for the users of the app, many social media apps use face recognition techniques to identify the person present in the uploaded picture for tagging purpose which makes it artificially intelligent [40]. Many other websites use face recognition techniques as its mandatory security feature and added it as the identification tool for their users. For example, Apple uses face ID to identify the users of its devices and added it as a security feature for its devices [7]. Many other agencies have widened the use of face recognition and uses it as a reliable tool of their security features for the identification verification services [10]. Many international airports use face recognition techniques to identify the travelers face with the biometric and facial data saved in the database of their citizens to keep the record of all their citizen who are on board and this process is totally automated which makes it artificially intelligent and reliable too. As the research in face recognition going deeper, the systems are becoming more and more accurate with reliable and accurate results which is also widening its applications parallel.

\section{Proposed Framework Methods}

In proposed framework we have considered basic theory and algorithm ideas of different subsystems used in proposed coalescence of image alignment and tagging in pose variations of human faces by Harris corner detection model and its application for pose-invariant face recognition system [37]. To solve the problem we have used three basic approaches for Pose Invariant Face Recognition (PIFR) [19, 20]. These approaches are as follows.

\subsection{Image Alignment}

Face alignment is a vision technology of computer, which is used to identify the structure of human face in the digital image geometrically. By knowing the location and size of the face, it determines the basic features of the face like nose eyes lips etc. and it starts adjusting a deformed image iteratively and finally determines the original image as much as possible and then matches its characteristics with the images saved in the database of known faces to extract the image best matching with the input image. Sometimes we see that the input face in the image is not aligned in a proper way to detect it as a human face from here the challenge for face recognition starts when the input image of human face is not detectable is human face images saved in the database. Fig. 2 shows the pose changes in 2D and in 3D in which face detection is achieved with help of image alignment process. Here we take image as input and detect face then we find fiducial point of the face and wrap it with pose and template is also used for face detection. With help of fiducial point we get the $2 \mathrm{D}$ and $3 \mathrm{D}$ alignment of the image. 

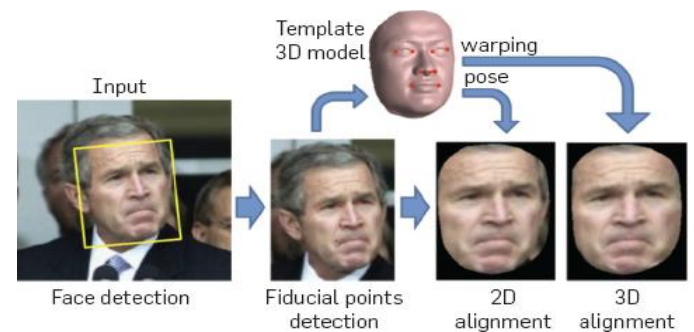

Fig 2:- Face detection with pose change

Reprint from: ACM computer association for computer machinery

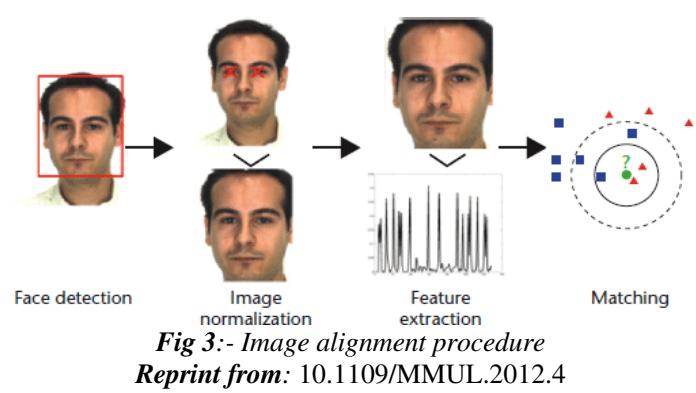

Now the image alignment techniques come in to picture where the algorithms detects the traces of human face and start aligning it iteratively to extract the maximum detailing of human face from the sun aligned image in the input Side and extracting the most matching image from the database to the unaligned image object in the input. Fig 3 shows the image alignment procedure from face detection to face matching [4]. Despite of many proposals on Image alignment, all those search methods aren't able to process the image alignment and to difficult to get the correct estimate of facial boundaries and true facial shape with pixel level of accuracy. Image alignment technology involves optimization of the image where the goal is matching the input image which is in deformed shape with the correct images as accurate as possible using the facial features detection and optimization processes [38]. In this optimization process of the input image, the accuracy of the system to find out the accurate image from the database matching with the input image totally based on the percentage of total number of features matched in both the images.

\subsection{Image Tagging}

When an input picture is being processed and the features extracted from the different processes are ready to be matched with the images available in the saved database, the system tries to extract the best matching image with the features of the input image to the system. Once the system finds the best suited database image with the input image, it adds a label to the input image with the other information available with the extracted image from the database. This information could be his/her name, gender, age, nationality etc. based on the information available and the programming of your system. This process is generally called image tagging where system automatically adds a tag with relevant information with the image.

\subsection{Pose Estimation}

Face recognition has vast application of deep learning. From phone unlocking to cameras on Airport, on criminal recognition in public areas, hi tech security applications etc. When you put together the facial recognition techniques with pose estimation technology, you get how much more powerful tool it is with more precise matches. The problem associated with this is the face of the human, which is a 3-D image, it can move or rotate in all three axis with some movement limitation [1]. In face pose estimation field, we know these rotations and movements as roll, pitch and yaw, as shown in the Figure 4 below:

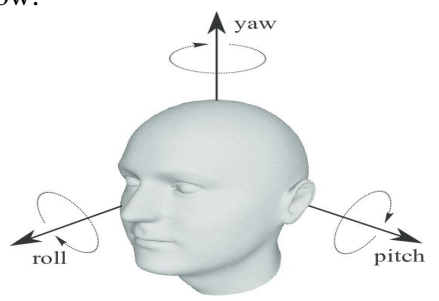

Fig 4:- Image shows face detection with pose change
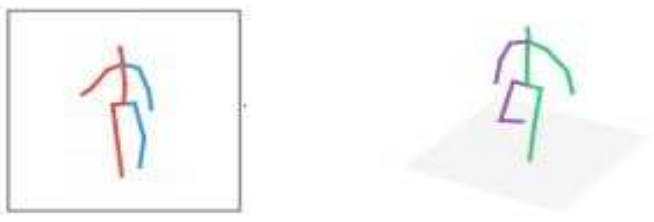

Fig 5:- Pose estimation in $2 D$ and in $3 D$ images. in $x, y$ and $z$ coordinates having pitch, yaw and roll angles

As we can observe, the main problem with the system to recognize the input phase and match it with the faces saved in the database, is that the input image is a 3-D image which has three dimensional movements in all the three axis. Pose Estimation is a very common problem in Computer Vision system in which position and orientation of an object detected. Pose estimation is applied on both either on 2D images and 3D images. In Fig 5 pose estimation is divided in two ways $2 \mathrm{D}$ pose estimation calculate the location of body with their pixel value. On the other hand 3D pose estimation calculate on three plane $\mathrm{x}, \mathrm{y}$ and $\mathrm{z}$. full body with their pixel arrangement gives us the final output.

2D Pose Estimation - In this 2D Estimate pose changes in between $(\mathrm{x}, \mathrm{y})$ coordinates of the plane for each angle joint in RGB image.

3D Pose Estimation - In this 3D Estimate pose changes in between $(\mathrm{x}, \mathrm{y}, \mathrm{z})$ coordinates of the plane for each angle joint in RGB image. Application are also support to use pose estimation which includes gaming in which high pose estimation is calculated, second is animation in which we are fully depend on pose change calculation because in animation every object changes their position very fast. Recognition of activity change also support pose estimation.

\section{Overview Pose Invariant Face Recognition}

Pose in varying face recognition PIFR is a hot topic of research. Its' application portfolio is widening up manifolds and increasing. Basically, the pose invariant face recognition PIFR deals with the non-ideal input images with different poses which are not easy to be detected as a perfect face of a human $[13,15]$.

Challenges to Pose Invariant Face Recognition in $2 D$ and $3 D$ :- In the recent years, many researchers have work on the large pores face recognition problem, the popular 2-D image-based method achieved a great improvement. Still the system has not yet been made perfect to give hundred percent accurate results. This is because 2-D \& 3-D matching problem [11]. Now the problem arises that how to make a system with $100 \%$ precision which could only take two-dimensional image of a three-dimensional object. This problem could be overcome with the help of algorithms. 


\section{4. Experimental Result and Discussion}

In this Section we explain the proposed system and get the result by applying the different methods. Face recognition is very useful application of image analysis. The real challenge for developer to build a system which is $100 \%$ accurate, fully automatic as well as overcome all the limitation during implementation. Pose correction also one of the challenge in the system. Human minds are quite intelligent to recognize, memories as well as identifies the human face in every condition. In artificial recognition deal with thousands of faces in different conditions. The computers having pre decided memory having fix computational speed.

\subsection{Pose correction}

Capability to recognize face images with their limitation is very tuff task in front of the researchers [9]. Pose variation is a task in one of them. In computational calculation, recognition images of the faces under varying pose still remain an open research area for the developer. Face recognition technology is the technology in which face is to be detected having different positions with different lightning conditions. Images can be processed either in 2D or in 3D. They are real images or saved in database. It is are of three type (1) matching in real view (2) apply transformation on images space (3) transformation in feature space. Now pose correction with transformation as well as angles is to be calculated yaw, roll and pitch value with transformation. Here we have discuss about the pose of an object with is relatively depend on the position of the object and camera as well as orientation of the object with respect to the camera. Pose estimation is the computer vision technique in which you can change the pose of an object according to the camera or pose change of camera according to object [5]. Pose estimation problem solve to find pose of an targeted object according to the camera used in which location of 3D points of object as well as image projection in 2D. Fig 6 shows the pose estimation in 2D plane as well as in 3D plane. Pose estimation is calculated in two ways with 3D objects with respect to camera.

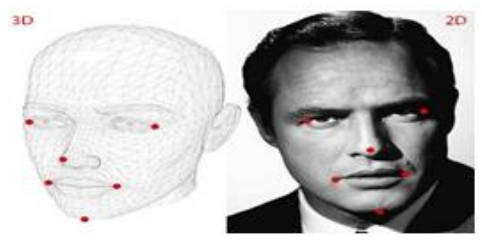

Fig 6:- Images shows with pose estimation in $2 D$ plane as well as in $3 D$ plane

i. Translation: Translation is the motion of the camera from its recent position to the new position in $3 \mathrm{D}$ location suppose in $3 \mathrm{D}$ plane the previous position of camera was $(X, Y, Z)$ so the new position in $3 \mathrm{D}$ is $\left(\mathrm{X}^{\prime}, \mathrm{Y}^{\prime}, \mathrm{Z}^{\prime}\right)$ this moment in position of camera is called translation. This is also called degree of freedom in 3 directions $\mathrm{X}, \mathrm{Y}$ or $\mathrm{Z}$. Translation is the vector quantity in which position changes from $\left(X^{\prime}-X, Y^{\prime}-Y, Z^{\prime}-Z\right)$. Translation means moving of an object and cover some distance either in right or in left direction in a plane.

ii. Rotation: Rotation is the moment of the camera in three axis called X, Y and Z. in rotation we get 3 degree of freedom. Rotation explains in many ways. Euler angles called roll. Pitch and yaw by $3 \times 3$ matrix rotation. Rotation is the thing in which axis of the image is fixed but point of rotation is in degrees from 1 to 360 degree in a plane. So pose estimation in $3 \mathrm{D}$ object calculated the 3 number in translation and 3 numbers in rotation. Total resultant is 6 numbers.

1. 2D coordinates: Few points from $2 D$ planes with $X$ and $Y$ coordinates $2 \mathrm{D}(\mathrm{X}, \mathrm{Y})$, some points from $2 \mathrm{D}$ plane suppose we consider an object as face so in 2D plane we chose corner of the eyes, nose tip, mouth corner points etc. by using landmark detector of face called facial landmark detector. These all are the frontal facial features with corner detection. Here we required nose tip, the chin, and the left corner of the left eye, the right corner of the right eye, the left corner of the mouth, and the right corner of the mouth. 2. 3D coordinates: in this location of the object is calculated in $3 \mathrm{D}$ plane of the same location of 2D features facial points. Here first a fall we need the position of the head as an object. We needs some arbitrary points like

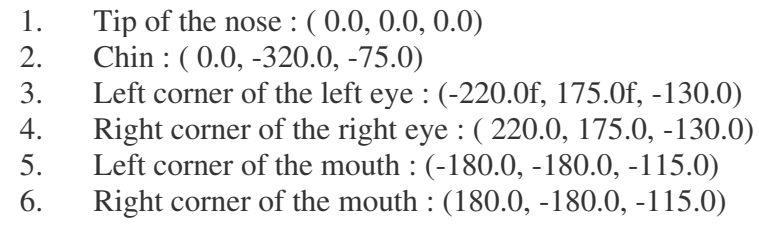

3. Camera parameters: In this we fully focused on camera location and focal length of it. Except the image camera location is also important to calculate the exact position of the object because if the location of camera is not set then no use to calculate the pose estimation of an object in image.

\section{Proposed Algorithm for Pose Estimation}

In pose estimation algorithm three coordinates are being in consideration. If we are going back in 1841 first known algorithm was introduced which is quite useful related to this field. Here we

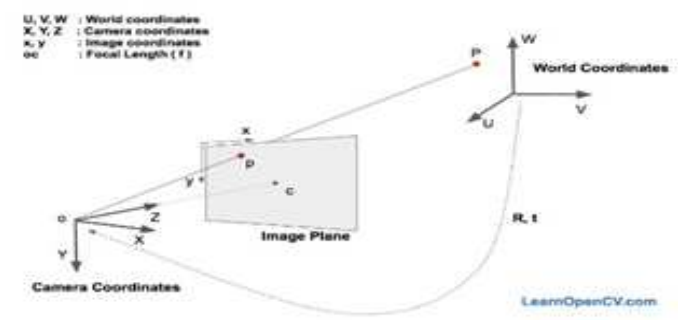

Fig 7:- Pose estimation in $3 D$ by using open $C V$ Calculations having world coordinates

are consider the object as a face which having various facial features. In pose calculation estimation we first calculate the translation as well as rotation that is pose calculation here $3 \mathrm{D}$ points of the camera is also know to us. The 3D points in camera coordinates can be projected onto the image plane (i.e. system of coordinate of image) with the help of the intrinsic parameters of the camera (focal length, optical centre etc.). In fig 7 pose estimation in 3D by using open CV calculations having world coordinates [38, 39]. In the figure above, $O$ is the camera centre and plane shown in the figure is the image plane. Here finding the equations of the projection $\mathrm{P}$ of the $3 \mathrm{D}$ point $\mathrm{P}$ onto the image plane. Let's suppose the location of world coordinates whose locations are $(\mathrm{U}, \mathrm{V}, \mathrm{W})$ in $3 \mathrm{D}$ plane here rotation $\mathbf{R}$ with $3 \times 3$ matrix and translation representation by $\mathbf{t}$ with matrix $3 \times 1$ vector with world coordinates with camera coordinates also in consideration. Now calculation of location $(\mathrm{X}, \mathrm{Y}, \mathrm{Z})$ with point $\mathrm{P}$ in camera coordinates with help of following equation 


$$
\begin{array}{llcc}
X & U & X & U \\
Y=\mathrm{R} & V+\mathrm{t} & =Y=[\mathrm{R} \mid \mathrm{t}] & V \\
Z & W & Z & W
\end{array}
$$

In expand form the above equation looks like

In linear algebra corresponding sufficient number of points which is location $(\mathrm{X}, \mathrm{Y}, \mathrm{Z})$ and $(\mathrm{U}, \mathrm{V}, \mathrm{W})$, the above linear equation where $\mathrm{r}_{\mathrm{ij}}$ and $\left(\mathrm{t}_{\mathrm{x}}, \mathrm{t}_{\mathrm{y}}, \mathrm{t}_{\mathrm{z}}\right)$ are not known. So the new equation will be

$$
\begin{array}{lrllll}
X & r_{00} & r_{01} & r_{02} & t_{x} & U \\
Y=r_{10} & r_{11} & r_{12} & t_{y} & V \\
Z & r_{20} & r_{21} & r_{22} & t_{z} & W
\end{array}
$$

We know $(\mathrm{X}, \mathrm{Y}, \mathrm{Z})$ only up to an unknown scale, and so simple linear system is on working. When the face of a human is in picture, yaw is the angle of moving head of the human from left to right side that means $\mathbf{y}$ - axis rotation, next is pitch that explains moving head of the moving human face from up to down means $\mathbf{x}$ - axis rotation last is roll the tilt angle means $\mathbf{z}-$ axis rotation. Which has been shown in Fig 8(a). In Fig 8(b) head pose rotation angle shows yaw roll and pitch angle with their coordinate axis $\mathrm{X}, \mathrm{Y}$ and $\mathrm{Z}$. and features are considered accordingly. In Fig 8(c) diagram moment of head according to estimated angles during pose transformation. In face recognition moment of head pose estimation in very tedious task because human face analysis is very easy for human but for computational calculation it's very minute calculation. In several computer vision system head position estimation is calculated as a first step now face expression, facial feature recognition and many more estimation is used at the time of pose invariant technology. Pose estimation is a process to find face orientation for an image which is either real image, 2D image or 3D image.

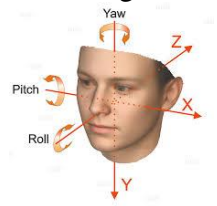

(a)

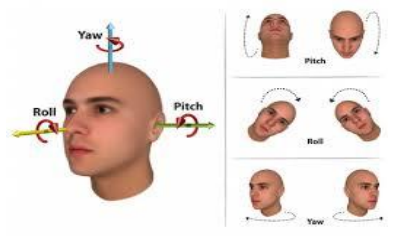

(b)
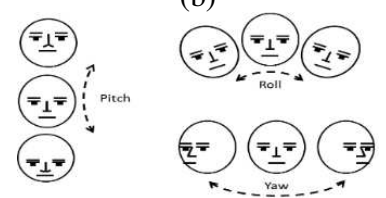

(c)

Fig 8:- (a) Head position according to yaw roll and pitch angle (b) Position of head according to head movement angles (c) Moment of head according to estimated angles during pose transformation.

Source: (a) Reprinted from [1]

Source: (b) Reprinted from [2]

Source: (c) Reprinted from [3]

In this section we summarize our nature inspired techniques for pose invariant face recognition (PIFR) [32]. The pseudocode of our

\begin{tabular}{|c|c|c|c|c|}
\hline $\begin{array}{r}S . \\
N o .\end{array}$ & $\begin{array}{l}\text { Image } \\
\text { naming }\end{array}$ & $\begin{array}{l}\text { Description of } \\
\text { Jmage }\end{array}$ & \multicolumn{2}{|c|}{ Pose Angle value } \\
\hline \multirow{3}{*}{1.} & \multirow{3}{*}{ face1.Jpg } & & Estimated Roll: - & 8.023286 \\
\hline & & & Estimated Pitch: - & 6.8465953 \\
\hline & & & Estimated Yaw: - & 7.1542964 \\
\hline \multirow{3}{*}{2.} & \multirow{3}{*}{ face2.jpg } & & Estimated Roll: - & -1.7462399 \\
\hline & & & Estimated Pitch: - & -1.9606336 \\
\hline & & & Estimated Yaw: - & 54.25557 \\
\hline \multirow{3}{*}{3.} & \multirow{3}{*}{ face3.jpg } & & Estimated Roll: - & -1.3936299 \\
\hline & & & Estimated Pitch: - & -23.063553 \\
\hline & & & Estimated Yaw: - & -21.68503 \\
\hline \multirow{3}{*}{4.} & \multirow{3}{*}{ face4.jpg } & & Estimated Roll: - & -3.8294692 \\
\hline & & & Estimated Pitch: - & -12.123642 \\
\hline & & & Estimated Yaw: - & -53.27958 \\
\hline \multirow{3}{*}{5.} & \multirow{3}{*}{ face5.jpg } & & Estimated Roll: - & 0.3337736 \\
\hline & & & Estimated Pitch: - & -1.8053052 \\
\hline & & & Estimated Yaw: - & 43.618565 \\
\hline \multirow{3}{*}{6.} & \multirow{3}{*}{ face6.jpg } & & Estimated Roll: - & 0.5642315 \\
\hline & & & Estimated Pitch: - & -11.203888 \\
\hline & & & Estimated Yaw: - & -43.178936 \\
\hline \multirow{3}{*}{7.} & \multirow{3}{*}{ face7.jpg } & & Estimated Roll: - & 0.70199084 \\
\hline & & & Estimated Pitch: - & -7.5902867 \\
\hline & & & Estimated Yaw: - & -46.741955 \\
\hline \multirow{3}{*}{8.} & \multirow{3}{*}{ face8.jpg } & & Estimated Roll: - & -1.0890148 \\
\hline & & & Estimated Pitch: - & -8.340223 \\
\hline & & & Estimated Yaw: - & -24.8332 \\
\hline \multirow{3}{*}{9.} & \multirow{3}{*}{ face9.jpg } & & Estimated Roll: - & -0.89014566 \\
\hline & & & Estimated Pitch: - & -8.099701 \\
\hline & & & Estimated Yaw: - & 33.578068 \\
\hline \multirow{3}{*}{10.} & \multirow{3}{*}{ face10.jpg } & & Estimated Roll: - & -3.7073011 \\
\hline & & & Estimated Pitch: - & -5.659264 \\
\hline & & & Estimated Yaw: - & 15.032032 \\
\hline \multirow{3}{*}{11.} & \multirow{3}{*}{ face11.jpg } & \multirow{3}{*}{$k=1$} & Estimated Roll: - & 4.0573435 \\
\hline & & & Estimated Pitch: - & 7.9625807 \\
\hline & & & Estimated Yaw: - & 46.737907 \\
\hline \multirow{3}{*}{12.} & \multirow{3}{*}{ face12.jpg } & & Estimated Roll: - & 1.0820476 \\
\hline & & & Estimated Pitch: - & 6.366044 \\
\hline & & & Estimated Yaw: - & 11.600706 \\
\hline
\end{tabular}

approach. In table 1 take twelve images of faces which is the combination of having different poses left right and front. Represent the value of pitch, yaw and roll having axis $\mathrm{X}, \mathrm{Y}$ and $\mathrm{Z}$ respectively. Calculate the value on each plane called as pose angle values. Compare all the values with all the images and draw graph accordingly. Which shows the difference in computation calculation according to pose changes during face recognition.

Table 1: Images with Angle Value

Approaching the PIFR techniques from graphical representation, which involves direct and indirect involvement of face changes computational calculation. In Fig. 9 the values of roll, pitch and yaw 
along the value on $\mathrm{X}, \mathrm{Y}$ and $\mathrm{Z}$ axis have been shown, which are having different poses right pose images, left pose images and front face images with slight variation: Fig.9 1(a) shows value of Roll according to pose angles of different images, Fig.9(b) shows value of Pitch according to pose angles on different images, Fig.9(c) shows value of Yaw according to pose angles on different images, and Fig.9(d) explains the combine value of Pitch Yaw and Roll according to pose angles on different images.
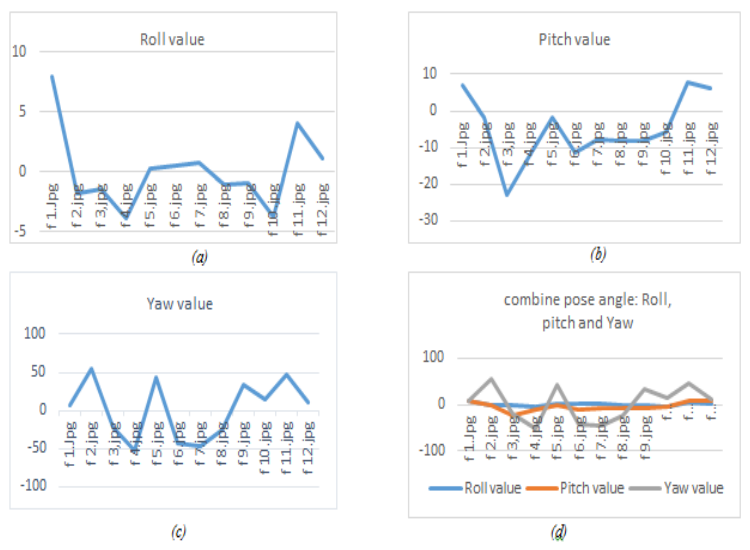

Fig.9 : (a) Roll value of pose angles of different images (b) Pitch value of pose angles of different images (c) Yaw value of pose angles of different images (d) combine value of pose angles of different images

\subsection{Experiment test of Face modeling}

Recognition of the face in normal conditions is easy by using

Face recognition algorithms, in which face database are used. On the other hand so many problem faced during face recognition face challenges like occlusion, lightening problem and pose variation is also one of them. Ability to recognize face under varying pose is a unique challenge for computer vision system. In past few decades, an unsolved problem in front of developer are Pose Invariant Face Recognition (PIFR). However, PIFR for real world application is used as passive biometric scientific knowledge for recognition of contrary subjects. Various methods of face recognition are present because of non-intrusive benefits of face recognition as a biometric method. Attention of face recognition is shifted towards PIFR research because of their growing benefits in this fields. It having speedy development for different novel approaches. Increment in PIFR publication in past years tell the story of success and importance so new insights developed for PIFR. In this section we are working on input image with their aligned image which covers all limitation of pose variation.

The above mentioned table and graph shows the value of pose change in images. In this section pose invariant properties is applied on images and correct the pose of face image. Applying transformation and alignment on the images then further calculation is applied on it. We performed experiment on real datasets images with different images having different pose angles similarity and difference is to be calculated and compared it with different poses. In Fig 10 alignments is to be applied on original image, which is fetched from query image. We have an image called input image now from image and recognize a face, which is called original image. When a pose system getting an original image all the processing is to be applied on original image. When an input picture is being processed and the features extracted from the different processes are ready to be matched with the images available in the saved database, the system tries to extract the best matching image with the features of the input image to the system. Once the system finds the best suited database image with the input image, it adds a label to the input image with the other information available with the extracted image from the database.

\begin{tabular}{|c|c|c|c|}
\hline $\begin{array}{c}\text { Image } \\
\text { naming }\end{array}$ & Input image & Original image & Aligned image \\
\hline face d4.jpg & & & \\
\hline Face d6.jpg & & & \\
\hline Face d2.jpg & & & \\
\hline Face d5.jpg & & & \\
\hline Face d3.jpg & & & \\
\hline Face d7.jpg & & & \\
\hline Face d8.jpg & & & \\
\hline
\end{tabular}

Fig 10:- Samples of image transformation having input images (query image), according to query image getting original image and alignment is to be applied on original image finally getting aligned image for further processing

\subsection{Discussion}

Based on the data table 2 in which 3D PIFR angles is to be calculated after calculating the PIFR angle graph 1 is drawn on the bases of value find on different images. Graph 1 shows the $\mathrm{x}, \mathrm{y}$ and $\mathrm{z}$ angle value in form of pitch, yaw and roll respectively. In graph $1(\mathrm{~d})$ combine result of each angle is represented. Value is generate according to the image is tilt or move from their front position. This angle is called pose angle. After calculating it in figure 10 input image is aligned on the basis of pose angle.

\section{Conclusion}

In this paper, we have proposed a natural inspired algorithm for PIFR (Pose Invariant Face Recognition). With relevant results, which is based on BPSO technique the relevant images have been considered for pose variation. The non-relevant images have been labeled by its pose angles in $\mathrm{x}, \mathrm{y}$ and $\mathrm{z}$ axis, which are called yaw pitch and yaw. Every image having their own values on different axis compare it and represent it graphically. In next section by Appling alignment on different images having different poses images and correct it. Our method is easy to implement, it also applied on stored pose databases and gives better result. Extensive experiments on different real as well stored database with 2D/3D image dis similarity measure have shown the excellence of the proposed method over different latest approaches. 


\section{Abbreviations \\ PIFR: Pose Invariant Face Recognition}

2D \& 3D: 2-Dimentions \& 3-Dimentions

BPSO: Biogeographical Particle Swarm Optimization

NN: Neural Network

LFW Dataset: Labeled Faces in the Wild database

\section{Declaration:}

\section{-Availability of data and materials}

The dataset supporting the results in this article is LFW dataset which is Labeled Faces in the Wild (LFW) database of facial photographs specially designed for studying the problem of unconstrained face recognition. Which is mentioned with in the article and the additional images are the real time images.

\section{- Competing interests}

Interest of author is in the area of image processing and its applications and competency with the latest technological advancements.

\section{- Funding}

No funding has been provided in the present work.

\section{- Authors' contributions}

Both authors take part equally in the preliminary research and discussion of the work explained in this paper. All authors read and approved the final manuscript. Designed the framework and designed the detailing of the algorithm explained in this article. Both the authors read and approved the final result for this manuscript

\section{- Acknowledgements}

The authors thanks to editor and the reviewers for their helpful suggestions and valuable comments which help guidance for this article.

\section{- Authors' information}

Department of Computer Science \& Engineering, Uttrakhand Technical University, Dehradun-248007, Uttrakhand India Deepika Dubey

Department of Electronics \& Communication Engineering, Director, Birla Institute of Applied Sciences (BIAS), Bhimtal- 263136, Uttrakhand India Geetam Singh Tomar

\section{References}

1. Mikel Ariz, Jos J. Bengoechea, Arantxa Villanueva, Rafael Cabeza,"A novel 2D/3D database with automatic face annotation for head tracking and pose estimation," Computer Vision and Image Understanding, Volume 148, Pages 201-210, July 2016.

2. Guru Kumar Lokku, G. Harinatha Reddy, M. N. Giri Prasad, "Automatic Face Recognition for Various Expressions and Facial Details," International Journal of Innovative Technology and Exploring Engineering (IJITEE), , Volume 8, Issue 9S3, Pages 264-268, July 2019

3. D. Banerjee and K. Yu, "Robotic Arm-Based Face Recognition Software Test Automation," IEEE Access, volume 6, Pages 3785837868, 2018. DOI: 10.1109/ACCESS.2018.2854754

4. A. K. Jain, B. Klare and U. Park, "Face Matching and Retrieval in Forensics Applications," IEEE Multi Media, Volume 19, Issue. 1, Pages 20-20, Jan. 2012. DOI: 10.1109/MMUL.2012.4

5. K. Okada and C. von der Malsburg, "Pose-invariant face recognition with parametric linear subspaces," Proceedings of Fifth IEEE International Conference on Automatic Face Gesture Recognition, Washington, DC, USA, 2002, pages 71-76.

6. R. Gross, Jie Yang and A. Waibel, "Growing Gaussian mixture models for pose invariant face recognition," Proceedings 15th International Conference on Pattern Recognition. ICPR-2000, Barcelona, Spain, 2000, pages 1088-1091 volume 1.

7. D. Beymer and T. Poggio, "Face recognition from one example view," Proceedings of IEEE International Conference on Computer Vision, Cambridge, MA, USA, 1995, pages 500-507. DOI: 10.1109/ICCV.1995.466898

8. R. Gross, I. Matthews, J. Cohn, T. Kanade and S. Baker, "MultiPIE," 2008 8th IEEE International Conference on Automatic Face \& Gesture Recognition, Amsterdam, 2008, pages 1-8. DOI: 10.1109/AFGR.2008.4813399

9. A. Lanitis, C. J. Taylor and T. F. Cootes, "Automatic interpretation and coding of face images using flexible models," in IEEE Transactions on Pattern Analysis and Machine Intelligence, volume 19, issue 7, pages 743-756, July 1997. DOI: $10.1109 / 34.598231$

10. Yongsheng Gao and M. K. H. Leung, "Face recognition using line edge map," IEEE Transactions on Pattern Analysis and Machine Intelligence, volume 24, issue 6, pages 764-779, June 2002.DOI: 10.1109/TPAMI.2002.1008383

11. P. Sharma, R. N. Yadav and K. V. Arya, "Pose-invariant face recognition using curvelet neural network," IET Biometrics, Volume 3, Issue 3, Pages 128-138, Sept. 2014. DOI: 10.1049/ietbmt.2013.0019.

12. Wang D., Hoi S.C.H. and He Y, "An Effective Approach to Pose Invariant 3D Face Recognition," Computer Science Springer, Berlin, Heidelberg International Conference on Multimedia Modeling MMM 2011: Advances in Multimedia Modeling, Volume 6523, pages 217-228. DOI: https://doi.org/10.1007/978-3-64217832-0_21

13. X. Peng, X. Yu, K. Sohn, D. N. Metaxas and M. Chandraker, "Reconstruction-Based Disentanglement for Pose-Invariant Face Recognition," 2017 IEEE International Conference on Computer Vision (ICCV), Venice, 2017, Pages 1632-1641.

14. P. N. Belhumeur, J. P. Hespanha and D. J. Kriegman, "Eigenfaces vs. Fisherfaces: recognition using class specific linear projection," IEEE Transactions on Pattern Analysis and Machine Intelligence, volume 19, Issue 7, pages 711-720, July 1997.

15. X. Chai, S. Shan, X. Chen and W. Gao, "Locally Linear Regression for Pose-Invariant Face Recognition," IEEE Transactions on Image Processing, volume 16, Issue 7, pages 1716-1725, July 2007. DOI: 10.1109/TIP.2007.899195

16. X. Xiao and L. Li, "Face Recognition Based on the Probability Support Vector Machines," 2008 International Conference on Computer Science and Software Engineering, Hubei, 2008, Pages 907-910. DOI: 10.1109/CSSE.2008.869

17. I. K. Timotius, T. C. Linasari, I. Setyawan and A. A. Febrianto, "Face recognition using support vector machines and generalized discriminant analysis," 2011 6th International Conference on 
Telecommunication Systems, Services, and Applications (TSSA), Bali, 2011, Pages 8-10. DOI: 10.1109/TSSA.2011.6095397

18. Y. Li, "A Face Recognition System Using Support Vector Machines and Elastic Graph Matching," 2009 International Conference on Artificial Intelligence and Computational Intelligence, Shanghai, 2009, Pages 3-6. DOI: 10.1109/AICI.2009.149

19. Z. An, W. Deng, J. Hu, Y. Zhong and Y. Zhao, "APA: Adaptive Pose Alignment for Pose-Invariant Face Recognition," IEEE Access, Volume 7, Pages 14653-14670, 2019. DOI: 10.1109/ACCESS.2019.2894162

20. N. Dahm and Y. Gao, "A Novel Pose Invariant Face Recognition Approach Using a 2D-3D Searching Strategy," 2010 20th International Conference on Pattern Recognition, Istanbul, 2010, Pages 3967-3970.DOI: 10.1109/ICPR.2010.965

21. I. Channoufi, S. Bourouis, K. Hamrouni and N. Bouguila, "Deformable models based object tracking: Challenges and current researches," 2016 5th International Conference on Multimedia Computing and Systems (ICMCS), Marrakech, 2016, Pages 35-40.

22. H. Lee, H. Kwon, R. M. Robinson and W. D. Nothwang, "DTM Deformable template matching," 2016 IEEE International Conference on Acoustics, Speech and Signal Processing (ICASSP), Shanghai, 2016, Pages 1966-1970.

23. Yongsheng Gao and M. K. H. Leung, "Face recognition using line edge map," IEEE Transactions on Pattern Analysis and Machine Intelligence, Volume 24, Issue 6, Pages 764-779, June 2002. DOI: 10.1109/TPAMI.2002.1008383

24. J. Yu, Y. Rui, Y. Y. Tang and D. Tao, "High-Order Distance-Based Multiview Stochastic Learning in Image Classification," IEEE Transactions on Cybernetics, Volume 44, Issue 12, Pages 24312442, Dec. 2014. DOI: 10.1109/TCYB.2014.2307862

25. T. Maugey, A. Ortega and P. Frossard, "Graph-Based Representation for Multiview Image Geometry," IEEE Transactions on Image Processing, Volume 24, Issue 5, Pages 1573-1586, May 2015.DOI: 10.1109/TIP.2015.2400817

26. V. Blanz and T. Vetter, "Face recognition based on fitting a 3D morphable model," IEEE Transactions on Pattern Analysis and Machine Intelligence, Volume 25, Issue 9, Pages 1063-1074, Sept. 2003. DOI: 10.1109/TPAMI.2003.1227983

27. M. Baydoun and M. A. Al-Alaoui, "Enhancing Stereo Matching With Classification," IEEE Access, Volume 2, Pagse 485-499, 2014. A. U. Batur and M. H. Hayes, "Adaptive active appearance models," IEEE Transactions on Image Processing, Volume 14 Issue 11, Pages 1707-1721, Nov. 2005. DOI: 10.1109/TIP.2005.854473

28. M. C. Ionita, P. Corcoran and V. Buzuloiu, "On Color Texture Normalization for Active Appearance Models," IEEE Transactions on Image Processing, Volume 18, Issue 6, pages 1372-1378, June 2009. DOI: 10.1109/TIP.2009.2017163

29. R. C. G. Chehata, W. B. Mikhael and M. M. Abdelwahab, "Transform Domain Two Dimensional and diagonal Modular Principal Component Analysis for facial recognition employing different windowing techniques," 2013 IEEE 56th International Midwest Symposium on Circuits and Systems (MWSCAS),
Columbus, OH, 2013, Pages 1104-1107. DOI: 10.1109/MWSCAS.2013.6674845

30. C. Xiang, X. A. Fan and T. H. Lee, "Face recognition using recursive Fisher linear discriminant," IEEE Transactions on Image Processing, Volume 15, Issue 8, Pages 2097-2105, Aug. 2006. DOI: $10.1109 /$ TIP.2006.875225

31. X. Chai, S. Shan, X. Chen and W. Gao, "Locally Linear Regression for Pose-Invariant Face Recognition," IEEE Transactions on Image Processing, Volume 16, Issue 7, Pages 1716-1725, July 2007. DOI: $10.1109 /$ TIP.2007.899195

32. L. Lei, S. Kim, W. Park, D. Kim and S. Ko, "Eigen directional bitplanes for robust face recognition," IEEE Transactions on Consumer Electronics, Volume 60, Issue 4, Pages 702-709, Nov. 2014. DOI: 10.1109/TCE.2014.7027346

33. D. Chu, L. Liao, M. K. Ng and X. Wang, "Incremental Linear Discriminant Analysis: A Fast Algorithm and Comparisons," IEEE Transactions on Neural Networks and Learning Systems, Volume 26, Issue 11, Pages 2716-2735, Nov. 2015.

34. Jian Yang, A. F. Frangi, Jing-Yu Yang, David Zhang and Zhong Jin, "KPCA plus LDA: a complete kernel Fisher discriminant framework for feature extraction and recognition," IEEE Transactions on Pattern Analysis and Machine Intelligence, Volume 27, Issue 2, Pages 230-244, Feb. 2005. DOI: 10.1109/TPAMI.2005.33

35. X. Xiao and Y. Zhou, "Two-Dimensional Quaternion PCA and Sparse PCA," IEEE Transactions on Neural Networks and Learning Systems, Volume 30, Issue 7, Pages 2028-2042, July 2019. DOI: $10.1109 /$ TNNLS.2018.2872541

36. Pan H., Zhang Y., Li C., Wang H, "An Adaptive Harris Corner Detection Algorithm for Image Mosaic," Pattern Recognition. CCPR 2014. Communications in Computer and Information Science Springer, Berlin, Heidelberg, Volume 484, Pages 53-62, 2014. DOI: https://doi.org/10.1007/978-3-662-45643-9_6

37. D. Li, H. Zhou and K. M. Lam, "High-Resolution Face Verification Using Pore-Scale Facial Features," in IEEE Transactions on Image Processing, vol. 24, no. 8, pp. 2317-2327, Aug. 2015.

38. Naeem Iqbal Ratyal, Imtiaz Ahmad Taj, Usama Ijaz Bajwa, Muhammad Sajid, "3D face recognition based on pose and expression invariant alignment," in Computers \& Electrical Engineering, Volume 46, Pages 241-255, August 2015.

39. A. Asthana, M.J. Jones, T.K. Marks, K.H. Tieu, R. Goecke "Pose normalization via learned $2 \mathrm{~d}$ warping for fully automatic face recognition," Proceedings of British Machine and Vision Conference, 2011, Pages 1-11. DOI:10.5244/C.25.127

40. S.R. Arashloo, J. Kittler, W.J. Christmas, "Pose-invariant face recognition by matching on multi-resolution MRFS linked by super coupling transform," Computer Vision and Image Understanding, Volume 115, Issue7, Pages 1073-10832011.

41. S.R. Arashloo, J. Kittler, "Fast pose invariant face recognition using super coupled multiresolution Markov random fields on a GPU," Pattern Recognition Letter, Volume 48, Pages 49-59, 15 October 2014. DOI: https://doi.org/10.1016/j.patrec.2014.05.017 


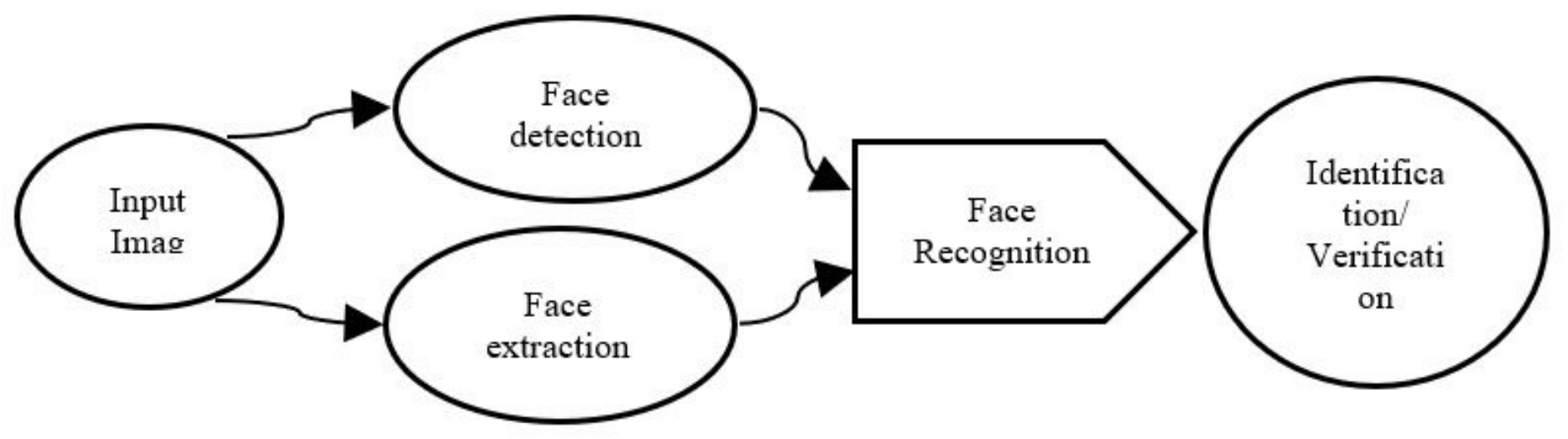

Figure 1

Face Detection and Recognition with Variation of faces

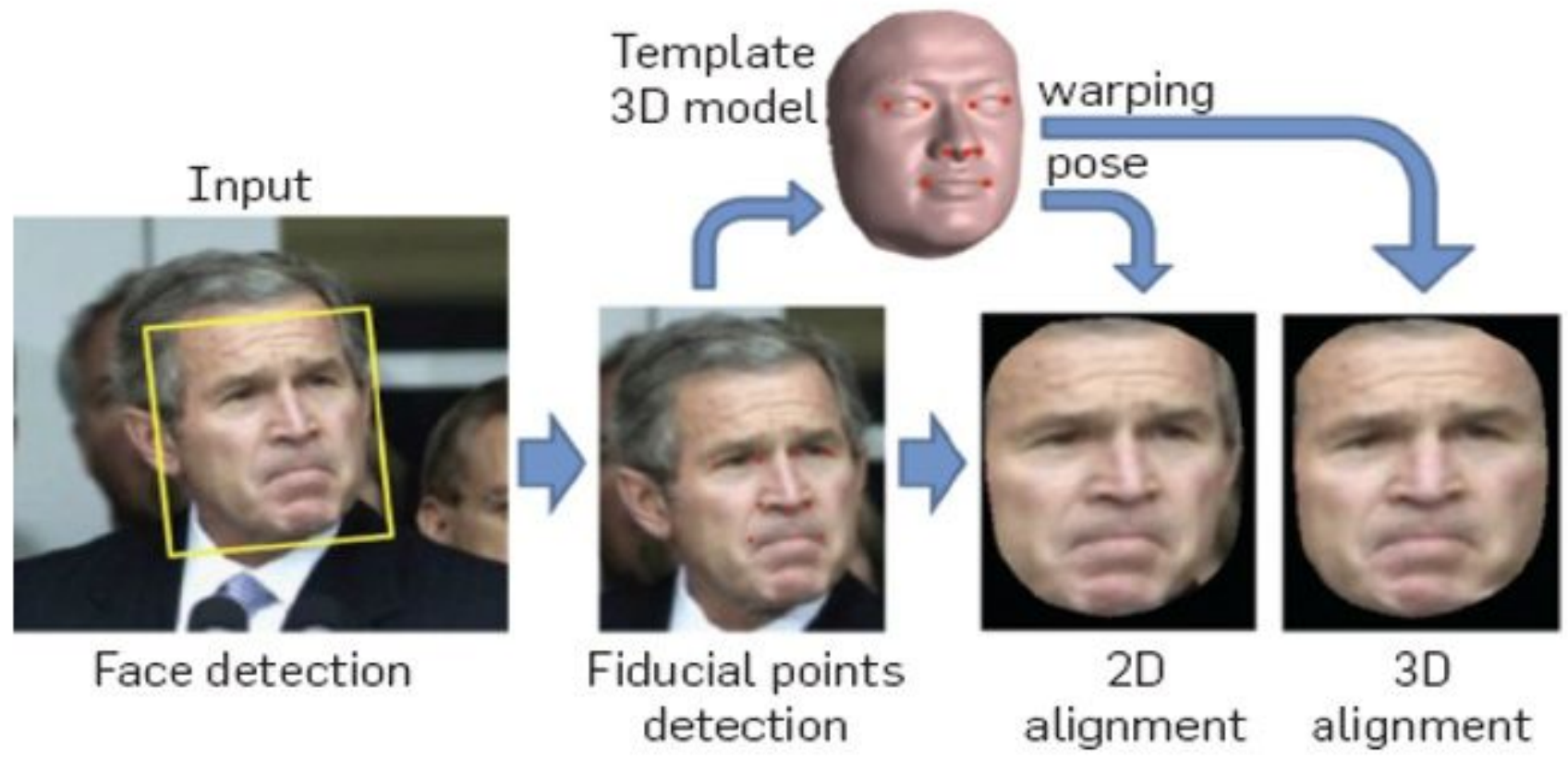

Figure 2

Face detection with pose change Reprint from: ACM computer association for computer machinery 


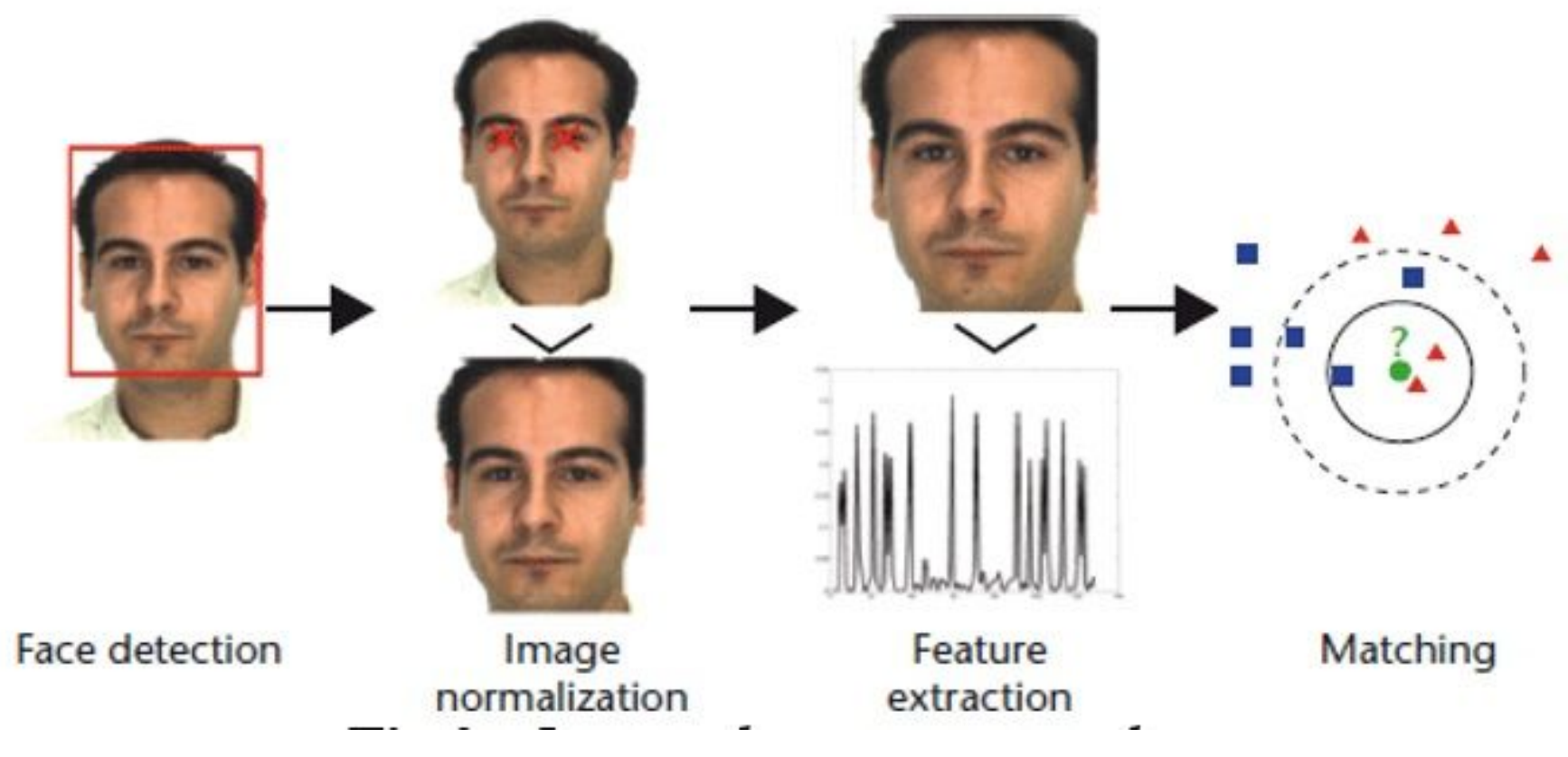

Figure 3

Image alignment procedure Reprint from: 10.1109/MMUL.2012.4

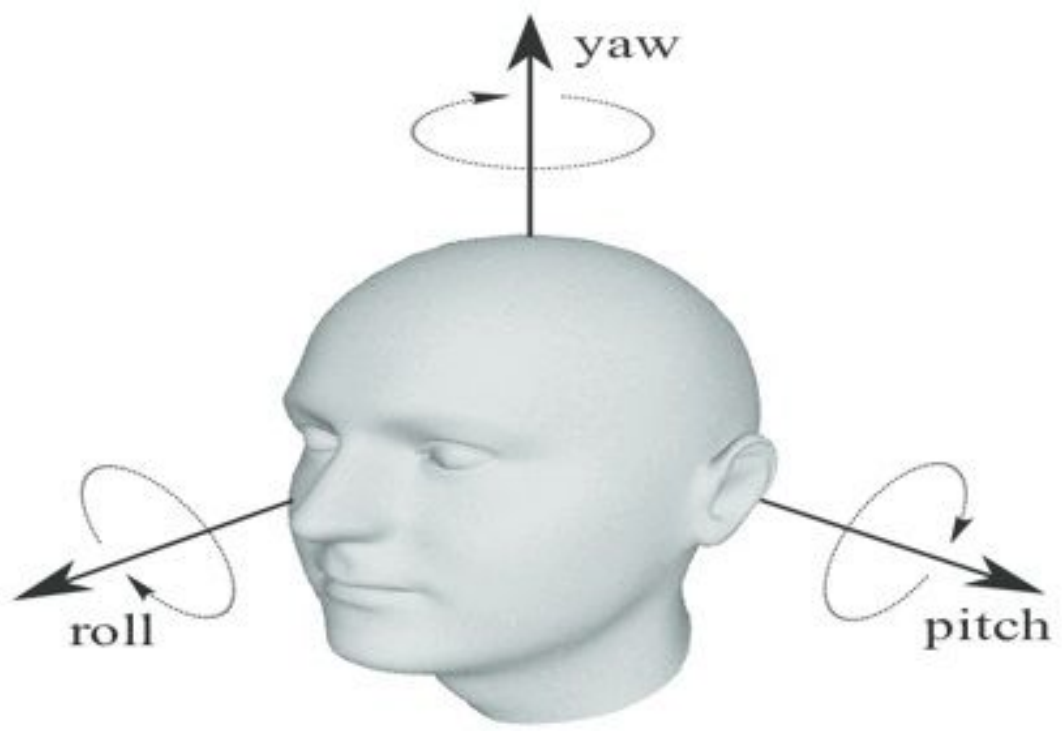

Figure 4

Image shows face detection with pose change 

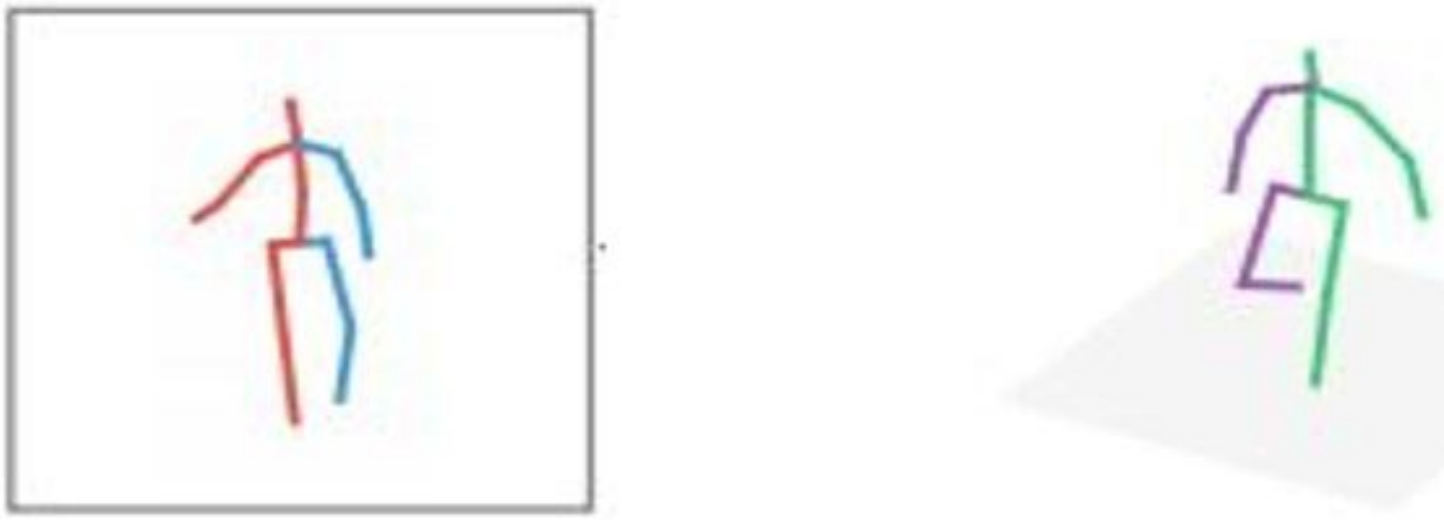

Figure 5

Pose estimation in 2D and in 3D images. in $\mathrm{x}, \mathrm{y}$ and $\mathrm{z}$ coordinates having pitch, yaw and roll angles

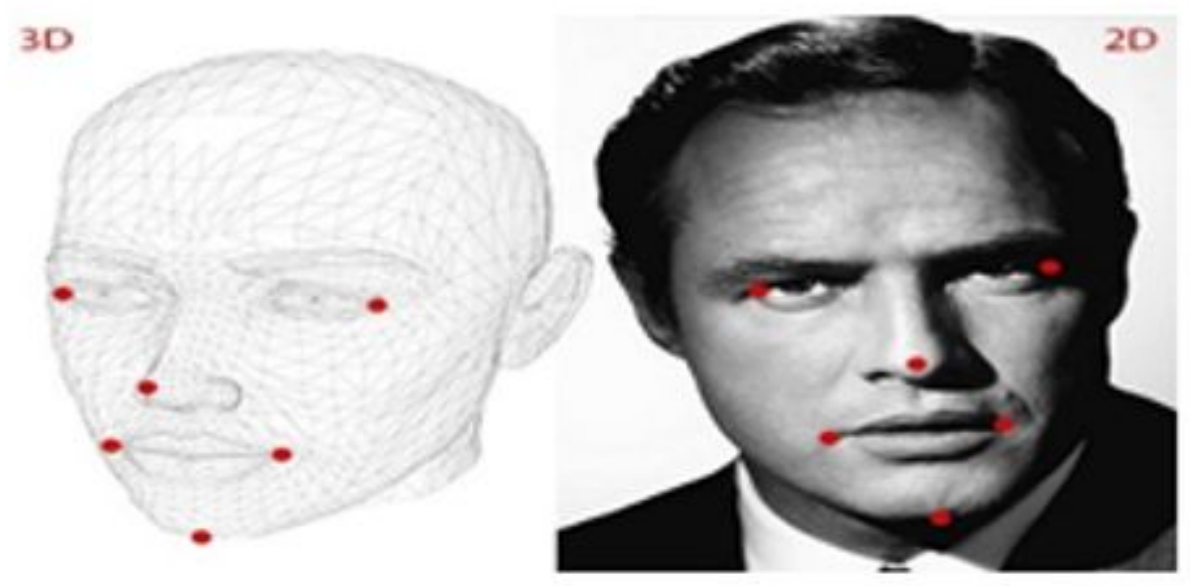

Figure 6

Images shows with pose estimation in 2D plane as well as in 3D plane 


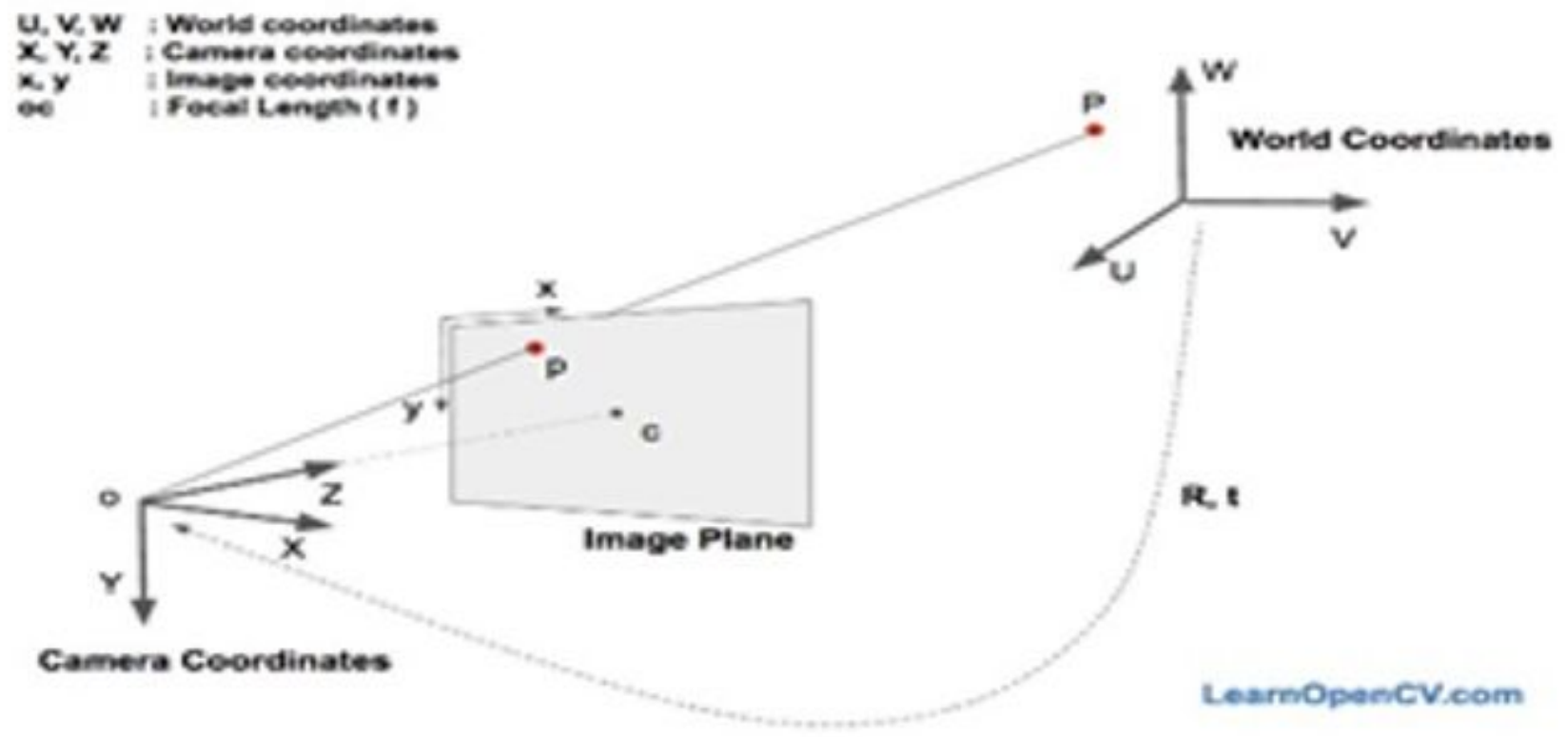

Figure 7

Pose estimation in 3D by using open CV Calculations having world coordinates 


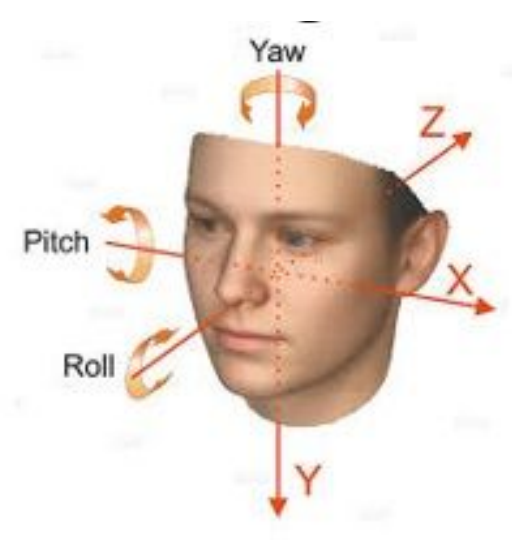

(a)

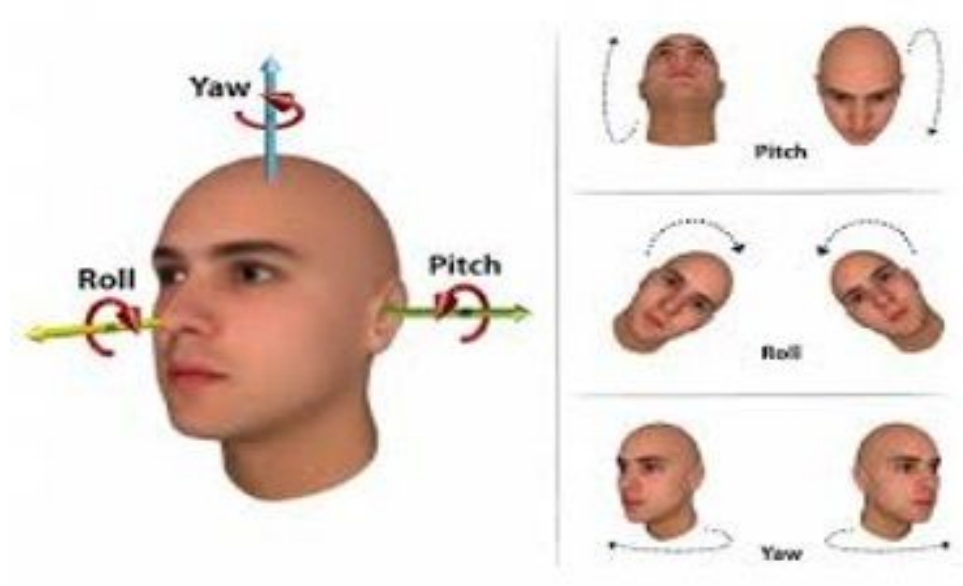

(b)
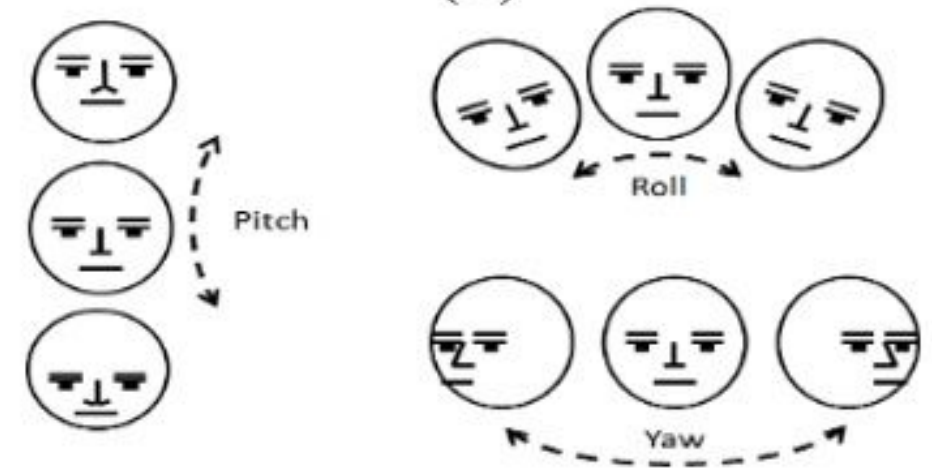

(c)

Figure 8

(a) Head position according to yaw roll and pitch angle (b) Position of head according to head movement angles (c) Moment of head according to estimated angles during pose transformation. Source: (a) Reprinted from [1] Source: (b) Reprinted from [2] Source: (c) Reprinted from [3] 


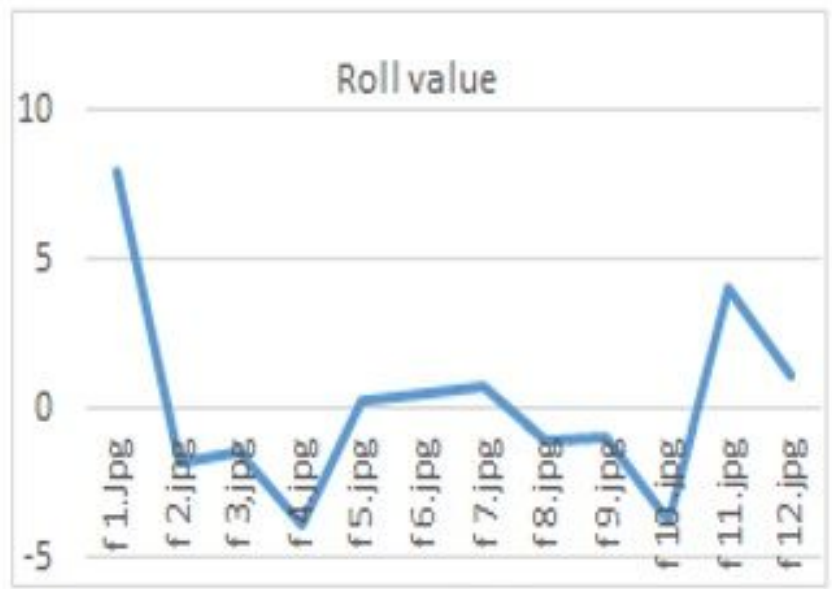

(a)

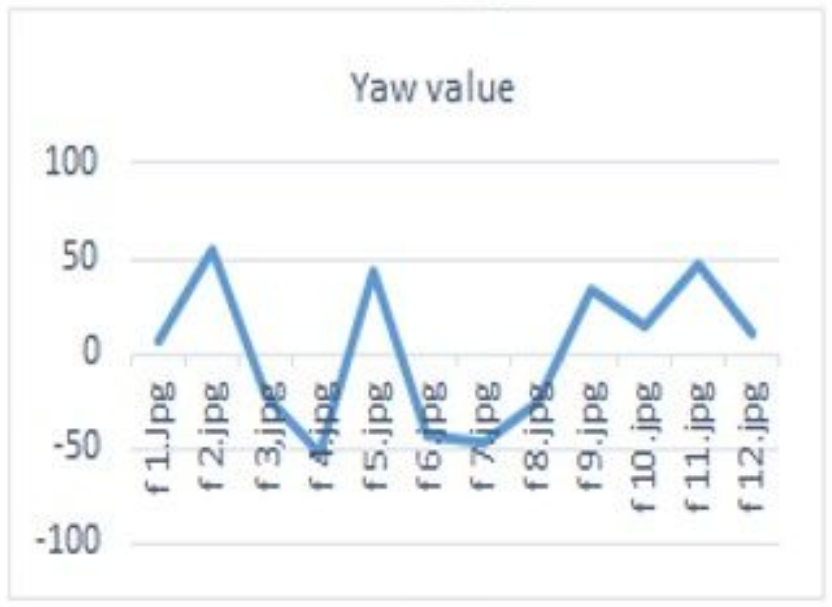

(c)

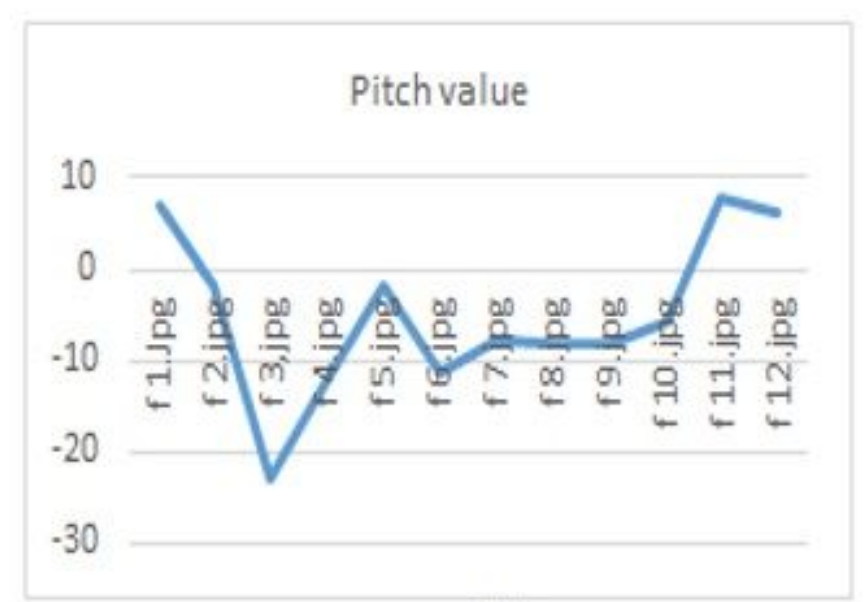

(b)

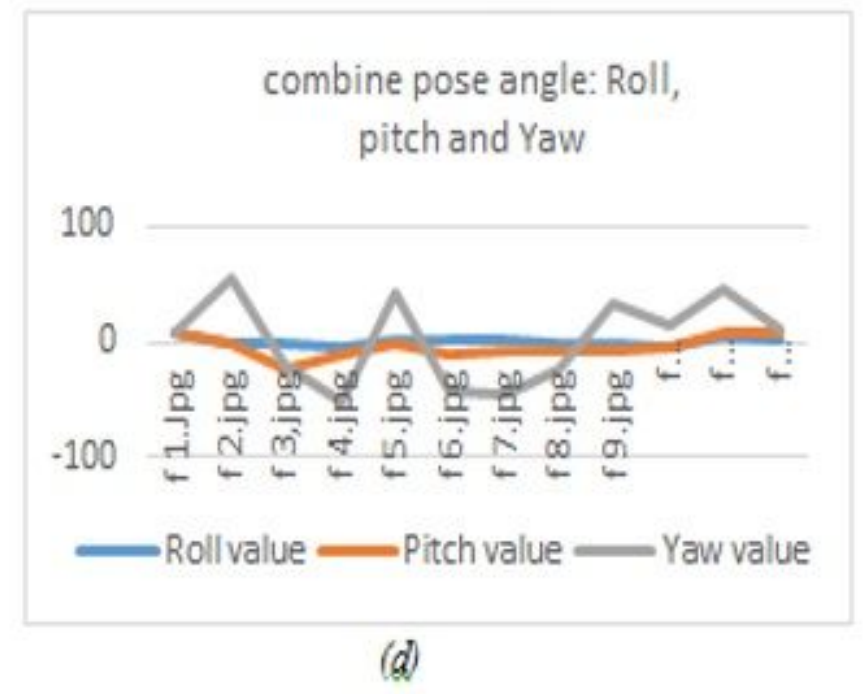

Figure 9

(a) Roll value of pose angles of different images (b) Pitch value of pose angles of different images (c) Yaw value of pose angles of different images (d) combine value of pose angles of different images 


\begin{tabular}{|c|c|c|c|}
\hline $\begin{array}{c}\text { Image } \\
\text { naming }\end{array}$ & Input image & Original image & Aligned image \\
\hline face d4.jpg & & & \\
\hline Face d6.jpg & & & \\
\hline Face d2.jpg & & & \\
\hline Face d5.jpg & & & \\
\hline Face d7.jpg & & & \\
\hline
\end{tabular}

Figure 10

Samples of image transformation having input images (query image), according to query image getting original image and alignment is to be applied on original image finally getting aligned image for further processing 